\title{
Atom Optics with Diffractive Structures
}

\author{
0. Carnal and J. Mlynek
}

Fakultät für Physik, Universität Konstanz, Germany

\author{
Experiments demonstrating the feasiblity of using nanoscale diffractive structures as optical \\ devices for atoms herald many promising applications of atom optics.
}

Atoms are usually thought of as hard spheres with a well-defined diameter, position and velocity. But at the beginning of this century, Louis de Broglie showed theoretically that they possess both particle and wave characteristics: an atom with mass $m$ and velocity $v$ behaves like a wave with wavelength $\lambda=\hbar / m v$, where $h$ is Planck's constant. The wave nature of atoms was verified experimentally shortly afterwards. The de Broglie wavelength $\lambda$ is very small, being about $0.1 \mathrm{~nm}$ for helium atoms with the velocity of $1000 \mathrm{~m} / \mathrm{s}$ typically found in a gas reservoir at room temperature: it decreases for heavier and faster atoms.

An atom can therefore be thought of as a wavepacket of a characteristic size with oscillations inside the packet having a period equal to the de Broglie wavelength. Depending on the experiment being performed, either the particle or the wave nature is predominant. The fact that atoms behave like waves implies that one can build, in principle, optical elements for atomic waves instead of light waves.

Interest in optical elements to, for example, focus, image and split beams of atoms in the same way as photons arises for mainly two reasons. First, an "atom microscope" has the potential to achieve high spatial resolution (in the nanometre region) with minimal damage to the surface under investigation since atoms are uncharged and can have kinetic energies below $10 \mathrm{meV}$. Second, an interferometer using atoms instead of light allows one to perform fundamental experiments on quantum mechanics and gravitation which were not possible before.

\section{Microscopy}

The resolution of a microscope is limited by the wavelength of the particles employed: it is impossible to resolve structures smaller than half the wavelength $(\approx 0.5 \mu \mathrm{m}$

Olivier Carnal is on the research staff of the Physics Faculty, University of Konstanz, Postfach 5560, W-7750 Konstanz. He received his diploma in physics from the ETH Zürich in 1988 where he worked with Professor Mlynek before spending a year at the ENS, Paris. He moved to Konstance in 1990 and was awarded his Ph.D. in 1991. Dr. Carnal was awarded the Swiss Physical Society's 1992 Balzers Prize. Professor Jürgen Mlynek has been with the University of Konstanz since 1990 after spending four years as an Assistant Professor at the ETH Zürich. He studied at the University of Hannover where he received his diploma (in 1976), a Ph.D. and his Habilitation (in 1984). He won the German Physical Society's 1987 Physikpreis and 1992 Leibniz Prize. in the visible). This lower limit can be decreased dramatically using electromagnetic waves with much shorter wavelengths such as X-rays or electron waves in an electron microscope. The maximum resolution of an electron microscope is comparable to the size of an atom $(\approx 0.1-1 \mathrm{~nm})$. But one has to pay a high price for this immense resolution: electrons are charged so specimens soon become charged leading to image distortion. X-rays carry a very large energy so specimens are easily damaged. The ideal solution would be a microscope based on particles with both a low energy and a short wavelength. Atoms fulfill these conditions perfectly. For example, a helium atom with $v=1000 \mathrm{~m} / \mathrm{s}$ and $\lambda \approx 0.1 \mathrm{~nm}$ has a kinetic energy of roughly $20 \mathrm{meV}$ which is about 1000 times smaller than that of an electron, and $10^{6}$ times smaller than that of an X-ray photon with a comparable wavelength. The low energy ensures that the surface being investigated is treated very gently and it reduces the penetration depth of atoms into the base material to about one atom layer. Only the uppermost surface layer is probed with atoms and the usually less interesting underlying structures do not contribute to the image. Moreover, by preparing atoms so that they carry an electric or magnetic moment, one can selectively probe the electronic and magnetic properties of a surface. In view of the important opportunities for atom microscopy, for surface-sensitive microprobes and even for atom lithography, there is great interest in building imaging elements for atoms.

\section{Interferometry}

Another fascinating application of optical elements for atoms involves interferometers. Such devices, also best known in classical optics, split an incoming light beam into two spatially separated beams and then recombine the beams. In coherent beam splitting, splitting and recombination have to be performed in such a way that the phase, i.e., the position of maxima and minima of the two waves, is preserved. Changes to the positions of the wave maxima along one path can be detected very accurately by measuring differences in the overlap of the two beams after recombination.

The total intensity at the output of an interferometer therefore tells us something about the relative phase difference between the two paths. This property can be exploited in the following way: the phase of an atom is altered by passing the atom through a region with a different potential energy. Since the total energy of the particle is preserved, the change in potential energy causes a reduction in kinetic energy, and therefore in the atomic velocity, and that the wave maxima for one path arrives slightly later at the detector. An interferometer can in fact detect potential differences between the two paths which are $10^{10}$ times smaller than the kinetic energy of the atoms.

The positions of the maxima can be changed, for instance, by the interaction of atoms with external fields or with other particles. Since atoms have an internal structure which does not exist in electrons or photons they feel a totally different environment. Atom interferometers therefore have a number of unique potential applications which will be presented later.

\section{Optical elements}

Optical elements for the visible domain (beam splitters, mirrors, lenses, etc.) are familiar to all of us. They ave found in photographic cameras, microscopes and lasers, and can be easily realised by passing light through glass substra with a refractive index not equal to one and having a special shape and polished surfaces. The construction of optical elements for neutral atoms is considerably more difficult since atoms cannot pass through solid materials.

Both ions and electrons can be easily deflected and manipulated by electric or magnetic fields. Building optical elements for charged particles is therefore a wellestablished technology, as can be seen in the results obtained in storage rings, accelerators and electron microscopes. However, these methods do not work for neutral atoms. The situation seemed hopeless until the invention of the laser and the tremendous developments in microfabrication over the last two decades. Together they have opened up two promising possibilities, presently being intensively investigated in many laboratories around the world, for realising optical elements for atoms based on interaction with laser fields and on diffraction from microfabricated transmission structures [1-3].

Optical elements relying on atom-laser interactions employ atomic absorption and emission processes in a strong laser field. Each such process is combined with the absorption or emission of a photon. Since each photon carries momentum, the atom experiences a "kick" leading to a considerable change in its velocity if these processes occur very often. One can therefore 

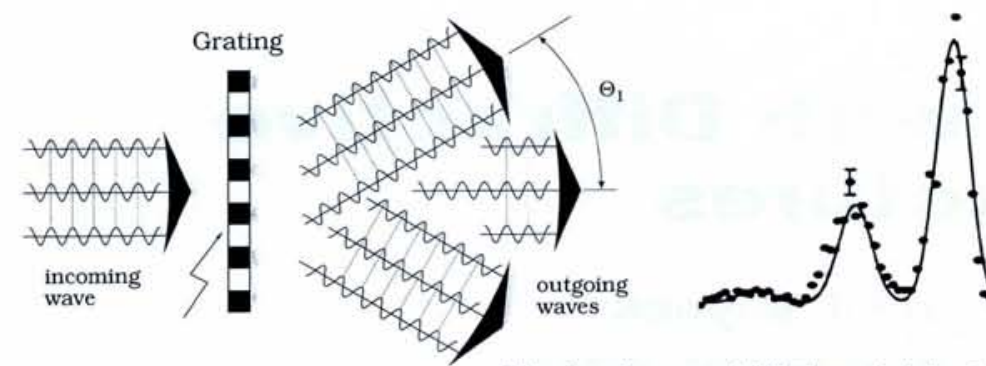

Fig. 1 - (a, upper left) The principle of a coherent beam splitter based on diffraction by a transmission grating. Partial waves emerging from the grating interfere constructively in directions $\theta n$.

|||||||||||||

(b, lower left) A scanning electron image of a grating for atom optics made from 100-500 $\mathrm{nm}$ thick gold foil by photolithogaphy. Note the precise, sharp edges to the approximately $100 \mathrm{~nm}$ wide slits [courtesy of Heidenhain Inc., Traunreut, Germany].

(c, right) Experimental results for a beam of metastable helium where the number of detected atoms is plotted as a function of the position perpendicular to the beam axis [4].

build lenses, mirrors and beam splitters for atoms by passing atoms through laser beams with properly shaped intensity profiles (see $[1,3]$ for a more detailed discussion of techniques).

We shall concentrate in this article on the technique that makes use of diffraction from microfabricated transmission structures. Since atoms have wave properties, they also experience diffraction from fine structures in the same way as light beams passing through gratings or small slits (see Fig. 1a). Many beams with different directions and intensities emerge from a diffracting element (Fig. 1b) and not just one beam. In the case of a grating with grating period $d$, this phenomenon can be explained as follows $[1,4]$ : an incoming beam (with wavelength $\lambda$ ) passes through one of the many different grating slits with equal probability. Since all partial beams have the same phase, the beams recombine constructively (all maxima are at the same place) in the forward direction $\left(\theta=0^{\circ}\right)$, and also in those directions given by $\sin (\theta n)= \pm n \lambda d d$ with $n$ $=1,2,3, \ldots$ where the path difference bet-

ween two neighbouring slits is exactly $\lambda$ The effect can be easily observed when the ratio $\lambda / d$ is close to unity.

Since all the diffraction orders have a well-defined phase relation, a diffraction grating is also called a coherent beam splitter. It can be used, for example, to build a beam separating element in an interferometer. To observe beam separation with atoms having a de Broglie wavelength of $0.1 \mathrm{~nm}$ one has to use extremely fine structures which must also be totally transparent at the slit positions. Such structures with dimensions down to $100 \mathrm{~nm}$ can be fabricated, for instance, out of very thin gold foils $(\approx 100$ $500 \mathrm{~nm}$ thick) using photolithographic techniques. They have extremely precise and sharp edges, as shown in Fig. 1b. Similar techniques can also be used to produce structures in the $100 \mathrm{~nm}$ range with silicon or silicon nitride films [5] and many groups in Germany, Japan and the United States are working to improve fabrication techniques. Since the diffraction process is insensitive to the atom's internal structure, these transmission structures can be used with all ato-

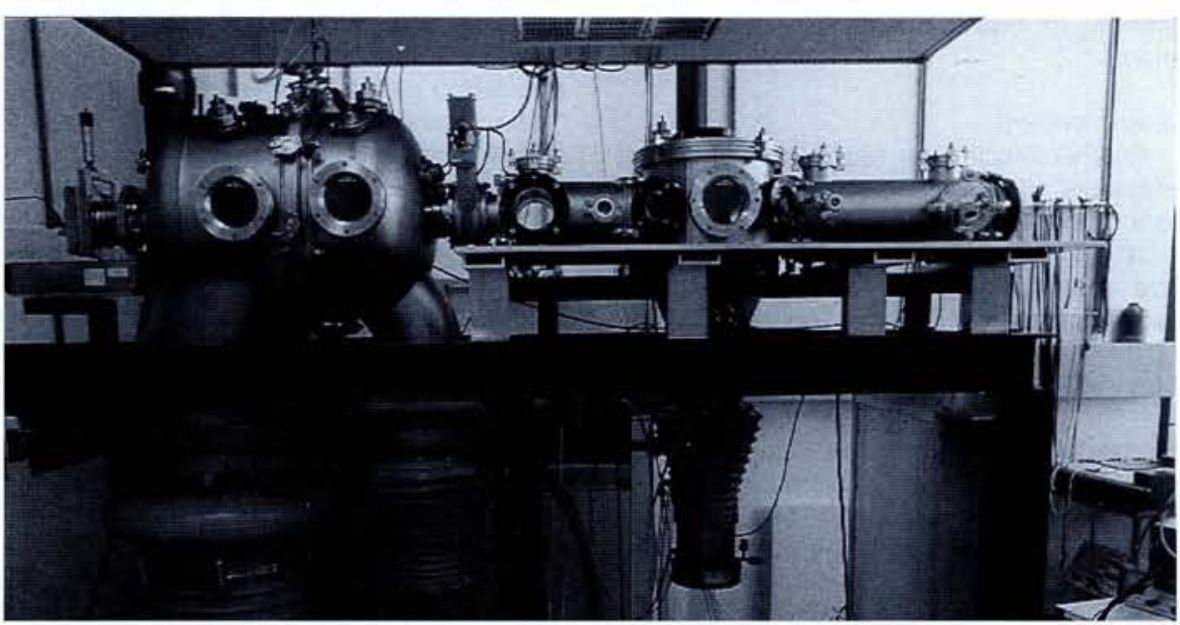

Fig. 2 - The experimental facility for experiments on atom optics. The atom source with its supersonic nozzle and electronic excitation is situated on the left. Metastable helium atoms travel horizontally to the right hand side of the vacuum tube where they are detected by a secondary electron emission. mic species and one easily changes from one atomic species to another without replacing the diffraction system.

\section{Atomic Source}

A prerequisite for experiments on optical elements for atoms is an intense source of atoms. We use a supersonic beam device (Fig. 2) which produces a very intense beam of metastable helium atoms (the two states prepared by electron beam excitation are $2^{1} \mathrm{~S}_{0}$ and $2^{3} \mathrm{~S}_{1}$, with a lifetime $\tau \approx 50$ seconds much longer than the transit time through the device). We also ensure that all the atoms have almost the same velocity. For supersonic beam expansions, where the primary pressure is so high (10-50 atm) that the mean free path of the atoms is much smaller than the diameter of the expansion nozzle $(10-25 \mu \mathrm{m})$, the velocities are compressed into a very narrow distribution around a thermal velocity owing to many-body collisions during the expansion. The narrow distribution means that the wavelength of each atom lies within a small range around a mean value corresponding to a light beam with a well-defined colour. Since the properties of optical elements depend of the wavelength, errors (aberrations) in an imaging system are minimised by employing a "monochromatic" beam of atoms of this type. Metastable atoms are preferred because they can be easily detected using a copper-beryllium surface with subsequent amplification of the emitted electrons in a secondary electron multiplier (SEM).

\section{Lenses}

A transmission structure made of gold has been used to build a lens for atoms [6]. It consists of a large $(\approx 130)$ number of concentric absorbing and transmitting zones, the diameters of which decrease as the square root of the number of the zone (see Fig. 3a). The gap between these absorbing gold rings therefore decreases with the distance from the centre of the lens. This geometry ensures that all the partial waves which would interfere destructively at a distance $L$ ' behind the zone plate are blocked. Only those waves which interfere constructively and therefore increase the intensity on the optical axis at a distance L' behind the lens are allowed to pass. If the source of atoms is situated a distance $L$ upstream of the lens (Fig. $3 b$ ), then $L$ and ' '' fulfill the lens equation $1 / L+1 / L^{\prime}=1 / f$, where $f$ is the focal length of the lens. Hence $f$ is proportional to the square of the innermost zone radius and inversely proportional to the wavelength of the atoms. Such a lens is called a Fresnel zone plate first examined by Lord Rayleigh as long ago as 1871.

We used for our experiments a zone plate with a diameter of $210 \mu \mathrm{m}$ and a diameter of the innermost zone of $18.5 \mu \mathrm{m}$. We installed a $50 \mu \mathrm{m}$ diameter wire in front of the lens [6] (a technique known as apodizing) in order to decrease the background atomic flux in the centre of the image plane and thereby increase image contrast. We once again performed experiments with metastable helium atoms which were detected with a SEM. The imaging properties of the zone plate were tested with single and double-slit 


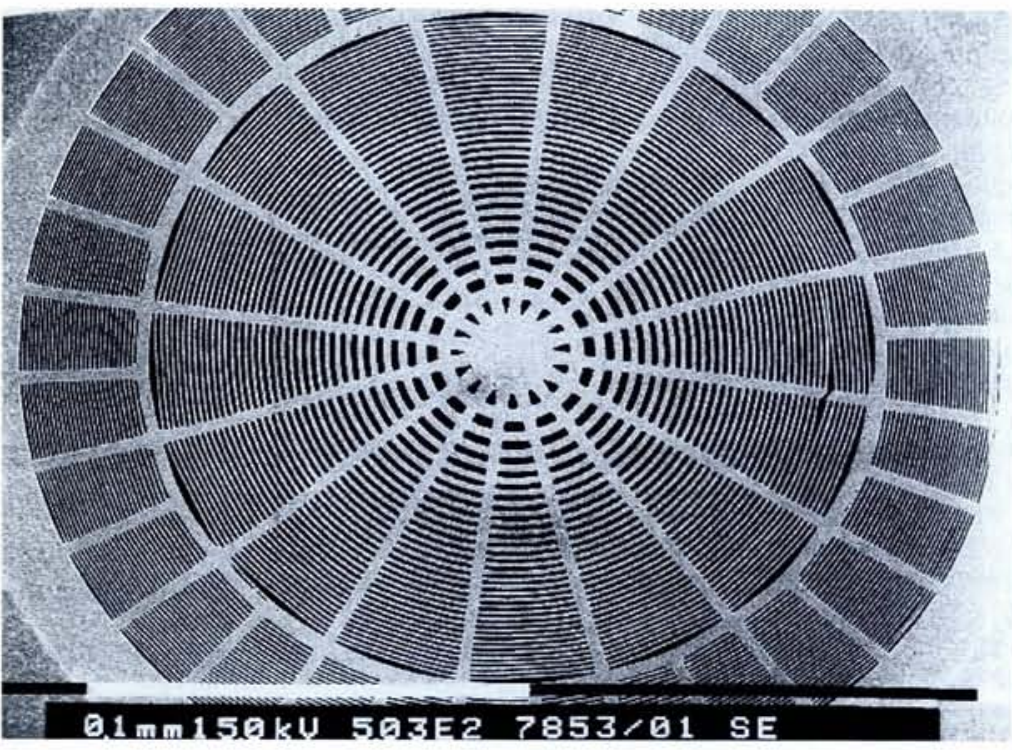

Fig. 3 - (a, left) A scanning electron image of the zone plate $(210 \mu \mathrm{m}$ diameter). The small crack on the right-hand side of the plate which formed during experiments did not affect imaging properties.

(b, upper right) A schematic illustration of the experimental setup for testing the zone plate. The dimensions are: $L=0.96 \mathrm{~m}, L^{\prime}=0.84 \mathrm{~m}$ and lens diameter $d=210 \mu \mathrm{m}$. The solid lines represent rays at the periphery forming the first-order image of the object structure.

(c, lower right) Image of a single slit and a double slit at an atomic wavelength $0.196 \mathrm{~nm}$. The solid lines are fitted to the data points. The slit dimensions noted in the insets showing the object structures are $s_{1}=10 \mu \mathrm{m}$ for the single slit and $d_{2}=49 \mu \mathrm{m}$ and $s_{2}=22 \mu \mathrm{m}$ for the double slit. BG denotes the detector background level (see [6] for details).

objects with slit dimensions in the $10 \mu \mathrm{m}$ range. The atom "images" were detected by scanning a $10 \mu \mathrm{m}$ slit across the image structure and detecting the helium atoms behind the detector slit with an SEM (see Fig. 3b). The experimental results are shown in Fig. 3c, where insets give the corresponding sizes of the object structures (to scale). The images show clearly that the atomic intensity in the detector plane is considerably increased with respect to the case without the lens. Second, in the case of the double slit as the object, the image size is smaller than the object size by a factor of $L^{\prime} / L=7 / 8$, which is exactly what one expects from a classical lens obeying the lens equation.

In order to eventually use such a lens in an atom microscope one has to increase its size so that more atoms can pass through the lens aperture, and decrease the focal length so that the optical system does not have unpractical large dimensions. The ratio of the focal length to the lens diameter is given by $s / \lambda$ where $s$ is the smallest slit structure on the zone plate. The size $s$ is presently limited to about $100 \mathrm{~nm}$ by the fabrication process and can only be decreased further using novel technologies. The advantage of this type of lens is the short interaction time of the atoms as they pass through ("thin" lens) - a feature that leads to reduced aberration compared to "thick" lenses based on the interaction with electromagnetic fields [3].

\section{An Atom Interferometer}

An atom interferometer was built by our group about 18 months ago [7]. The prin- ciple of our experiment, which was demonstrated for light in 1807 by Thomas Young, is very simple. Helium atoms pass through a small entrance slit and then through the upper or lower of two slits (separation w) positioned a distance $L$ further downstream (see Fig. 4a). Fig. 4b shows a scanning electron image of the double slit, the most critical element of our interferometer. The two, narrow, vertical, dark lines are the two $1 \mu \mathrm{m}$ wide slits. Two different paths (1 and 2) are thus possible for an atom travelling from the entrance to the detector slit. This double-slit arrangement is called an interferometer since it allows one to introduce something in between the two paths, without destroying the interference pattern at the detector. There are different positions in the detector plane where the two waves emer-

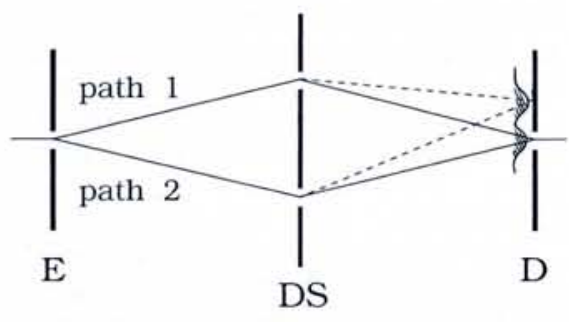

Fig. $4-(a$, left) A schematic illustration of an entrance slit $E$, a double slit DS and a detector slit $D$. Dark and bright fringes are observed in the detector plane with a period $\Delta x=L \lambda / d$. Dimensions are: $E$ to $D S-640 \mathrm{~mm}$; $D S$ to $D-640 \mathrm{~mm}$; $E$ and $D$ slits - $2 \mu \mathrm{m}$ wide. the double-slit interferometer. It consists of

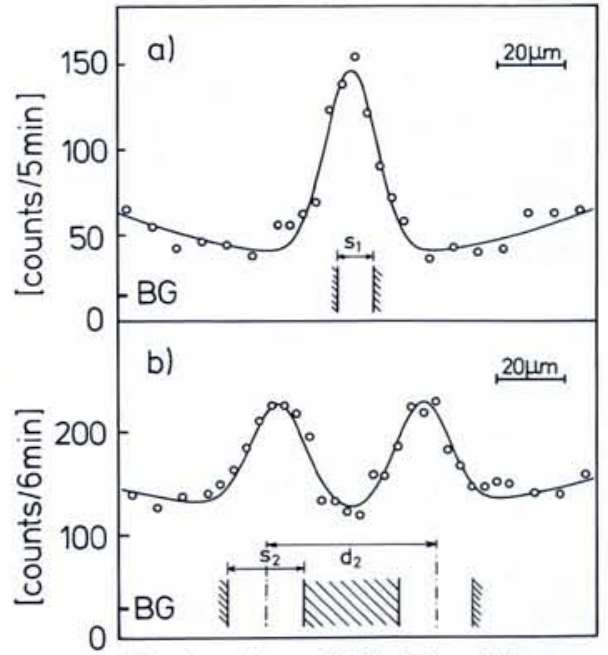

Detector Slit Position
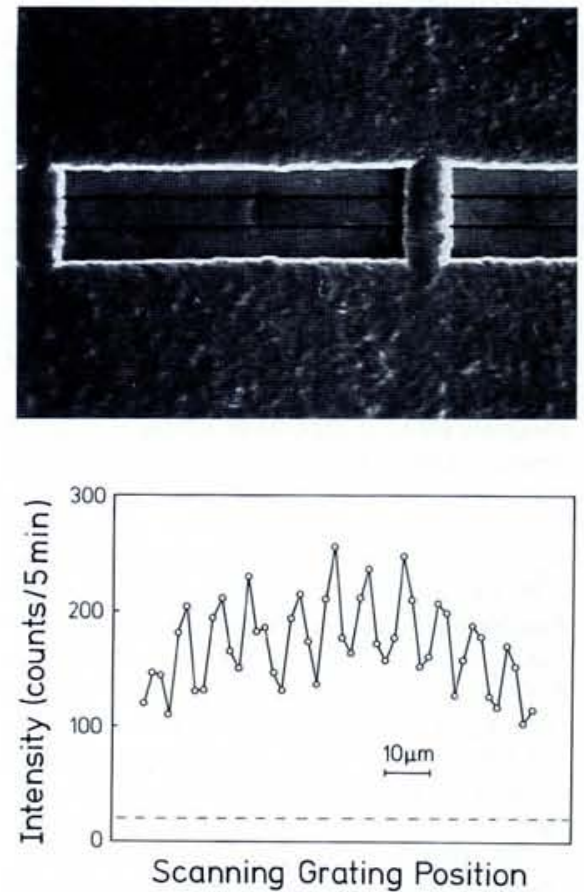

(b, upper right) Scanning electron images of the microfabricated double slit, with the slit structures in the horizontal direction and the support gratings in the vertical direction (slit width $1 \mu \mathrm{m}$; slit separation $8 \mu \mathrm{m}$; overall slit height $2 \mathrm{~mm}$ )

(c, lower right) Atom intensity profile, monitored with an $8 \mu \mathrm{m}$ period grating in the detector plane, as a function of the grating displacement. The dashed line is the detector background. The line connecting the experimental points is to guide the eye. 
ging from the two slits interfere constructively: they are located where the path difference between path 1 and 2 is exactly $\lambda$. An interference pattern with dark and bright fringes (fringe separation $\Delta x=L \lambda / w$ ) should arise on moving the detector slit in a direction perpendicular to the atomic beam axis.

The fringe pattern can be clearly seen in Fig. $4 \mathrm{c}$, where the distribution in the atomic intensity is monitored as a function of the detector position. To increase the total signal, a grating instead of the single slit has been used to detect the fringe pattern. The distance between two interference maxima in the experiment is close to $\Delta x=8 \mu \mathrm{m}$, as predicted by theory. If now a phase shift is introduced in one path, this interference pattern would move either to the left or to the right and the shift is a measure of the potential difference between paths 1 and 2 . Owing to the small slit dimensions, the detected signal rate is very low $(\approx 30-50$ atom counts $/ \mathrm{min}$ ) so the precision in measuring the phase shift is still rather limited. We can detect a phase shift of 0.3 when integrating the experimental results over $10 \mathrm{mi}$ nutes (i.e., one wave lags the other by about a tenth of a wavelength). The accuracy can be improved by increasing the atomic source intensity and the detector efficiency. In the meantime, different types of atom interferometers have been realised [1] using either micro-structures [8] or light fields as atomic beam splitters $[9,10]$.

\section{Further Developments}

Since atoms do not penetrate through solid, an atom microscope would always work in reflection whereby atoms strike a surface and reflected atoms are detected. A large area of the investigated surface under investigation can be irradiated with a plane atomic wave and the reflected atoms imaged using a zone plate. Alternatively, an atomic beam is focussed onto the surface and the total flux of reflected atoms or emitted electrons measured. Surface structures are resolved to a few nanometres in both cases. Atoms can be easily polarized, magnetized or prepared in different internal energy states with laser beams. This means that very different properties of the surface, such as the magnetization of a magnetic tape or surface roughness, can be observed with the same atom-optical system. Even though the resolution of the system will not reach those found in scanning tunnelling or electron microscopes, the fact that atoms have such small energies and can be prepared with many internal states will make this novel instrument an interesting tool for surface scientists. So while an atom microscope will never replace an electron microscope it can help obtain important complementary information.

Atoms are heavier, slower moving and have a much richer internal structure than other particles so it is useful to repeat interferometry experiments using atoms instead of, say, neutrons to give higher precision and to even allow measurements which were not possible before. Of practical interest is the observation of gravitational fields and of rotations of the interferometer frame [9-11]. Since the strength of the interaction of an atom with a gravitational field is pro- portional to the particle mass $m$, atomic waves are strongly affected by external field variations (the potential energy difference between the two paths is $\mathrm{mgd}$, where $g$ is the gravitational constant). This feature could be exploited, in principle, to detect mineral deposits which distort the local gravitational field. Moreover, the low velocity of atoms makes an atom interferometer very sensitive to rotations so it could eventually be used as a gyroscope (rotation sensor)

But for practical applications, the interferometer size (especially the beam separation) and the intensity of the atomic beam source have to be increased considerably. Atom-laser interactions can be used to decrease the divergence of the atomic beam and the velocity spread in the beam direction [12]. These laser cooling techniques also offer the possibility to decrease the atomic velocity so dramatically that the de Broglie wavelength becomes comparable to the wavelength of visible light, thereby facilitating experiments and increasing the sensitivity of the interferometer. In this context, a light-pulse atom interferometer has been used to measure $g$ with lasercooled sodium atoms in an atom "fountain" [10]. It is expected that an atom interferometer-based gravitometer of this type would attain an absolute accuracy of better than $10^{-10} \mathrm{~g}$.

Atom interferometers also make it possible to investigate interactions with magnetic and electric fields with high precision. For example, general tests of the charge neutrality of atoms may be feasible since an atom's response to these fields depends strongly on specially prepared internal states. Secondly, an atom interferometer allows one, in principle, to measure the intensity of a laser beam crossing the interferometer without destroying any photons in the laser beam [1]. Atom interferometers thus offer new and promising opportunities which would perhaps lead to presently unknown applications in fundamental research and sensor technology in the near future.

Besides potential applications in interferometry, microscopy or lithography, atom optics itself remains of much interest, both from the experimental and theoretical points of view. As one example, consider atom cavities: by using appropriate atom mirrors it should be possible to trap atoms in a cavity. There is even no need for two mirrors, because due to gravity and with very slowly moving atoms one can realise a cavity with only one single concave mirror facing upwards [13]. If the phase of the wave function can be preserved upon reflection, one would have resonant cavities, analogous to the Fabry-Perot cavities of usual optics, with perhaps more than one atom per mode.

\section{Conclusion}

This discussion of optical elements for atoms, based on diffraction by microfabricated Fresnel zone plates for focussing and double-slit Young arrangements for interferometry, involves just two of the many types of optical devices for atoms which can be conceived. It nonetheless illustrates that atom optics has developed within a few years into a whole new field with very promising opportunities.

\section{ACKNOWLEDGEMENT}

We are indebted to the Deutsche Forschungsgemeinschaft for support.

The present article is adapted from a text published this month in La Recherche.

[1] Special Issue of Appl. Phys. B 54 (May 1992): Eds: J. Mlynek, V. Balykin and P. Meystre.

[2] Pritchard D.E., "Atom Optics", Eds: J.C. Zorn and R.R. Lewiss; ICAP 12 (Ann Arbor, MI, 1991) 165.

[3] Balykin V.I. and Letokhov V.S., Physics Today (1989) 23.

[4] Carnal. O., Faulstich A. and Mlynek J., Appl. Phys. B 53 (1991) 88.

[5] Tennant D.M. et al., J. Vac. Sci. \& Tech. B 8 (1990) 1975; Pool R., Science 255 (1992) 1513.

[6] Camal O. et al., Phys. Rev. Lett. 67 (1991) 3231.

[7] Carnal O. and Mlynek J., Phys. Rev. Lett. 66 (1991) 2689.

[8] Keith D.W. et al., Phys. Rev. Lett. 66 (1991) 2693; Shimzu F., Shimizu K. and Takuma H., Jpn. J. Appl. Phys. 31 (1992) L436.

[9] Riehle F. et al., Phys. Rev. Lett. 67 (1991) 177.

[10] Kasevich M. and Chu S., Phys. Rev. Lett. 67 (1991) 181.

[11] Clauser J.F., Physica B 151 (1988) 262.

[12] "Laser Cooling and Trapping of Atoms", Special Issue of J. Opt. Soc. Am. B6 (1989), Eds.: S. Chu and C. Wieman.

[13] Wallis H., J. Dalibard J. and CohenTannoudji C., in [1].

\section{proFit, a powerful tool for analysis and graphical presentation of scientific and technical data on the Macintosh}

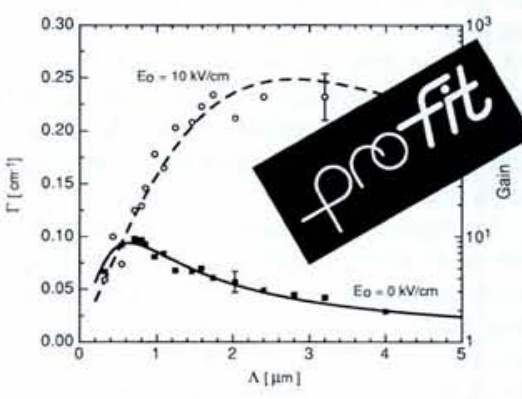

- professional graphical environment camera ready, high quality drawings highly interactive curve fitting

built-in set of standard functions

powerful definition syntax for custom functions

versatile possibilities for data processing full support of system 7

background operation

reasonable price

\section{QuantumSoft}

Postfach $6613 \cdot \mathrm{CH}-8023$ Zürich

Tel. (+41) 52/43 4174 • Fax (+41) 1/820 3836 